\title{
Single State Variational Calculations with applications to Large and Small Magnetic Fields
}

\author{
B L Burrows ${ }^{1}$, and M.Cohen ${ }^{2}$ \\ ${ }^{1}$ Mathematics Section \\ School of Creative Arts and Engineering \\ Staffordshire University, College Road, Stoke-on-Trent,ST4 2DE UK \\ e-mail:brian.burrows2@btopenworld.com \\ ${ }^{2}$ Department of Physical Chemistry \\ The Hebrew University of Jerusalem \\ Jerusalem 91904, Israel \\ e-mail:maurice@fh.huji.ac.il
}

31 January 2020

\begin{abstract}
Variational techniques to calculate estimates of bound-state energies and wave functions for single states are applied to simple electronic systems in magnetic fields. Explicit constraints to other state functions are not required. Our numerical energies compare favourably with results of other calculations, and the speed of our calculations is greatly enhanced by using a separable basis set and analytical integration.
\end{abstract}




\section{Introduction}

Many calculations of the effects of magnetic fields on atomic and molecular systems have concentrated on small field strengths, and have usually been performed in the framework of perturbation theory. Here, we treat large fields as well as small, and so resort to variational calculations suitable for discrete bound states. Details of our calculations are given in the following sections, together with a simple example that illustrates both the theory and the method of our calculations. Essentially, our choice of basis is tailored for each state treated, and contains a set of linear and non-linear parameters appropriate to each state studied. With these parameters fixed, we undertake an $N \times N$ matrix calculation of the lowest $\mathrm{N}$ states of the system. As is well-known, the eigenvalues of this matrix provide upper bounds to each of the states separately, and may be minimised with respect to all embedded parameters for each state separately $[1,2,3]$. Slightly different choices have been examined recently using a more complicated energy functional [4]. In the present calculations, a single nonlinear parameter suffices. We treat the atom $\mathrm{H}$, and the diatomic ion $\mathrm{H}_{2}^{+}$, in the latter case with the field parallel to the internuclear axis. Our results are compared with some earlier treatments $[5,6]$ and some more recent work $[7,8,9]$ which extend the traditional treatment to very large fields.

\section{Specification of a state variatiationally}

For any normalised real wave function $\psi$ and Hermitian $\mathrm{H}$ we have

$$
E=\frac{<\psi \mid H \psi>}{<\psi \mid \psi>}, \quad W=\frac{<(\psi+d \psi) \mid H(\psi+d \psi>}{<\psi+d \psi \mid \psi+d \psi>}
$$

then since $\psi$ is normalied

$$
W=\frac{E+2<d \psi|H \psi>+<d \psi| H d \psi>}{1+2<d \psi|\psi>+<d \psi| d \psi>}
$$

so that

$$
W=E+\frac{2<d \psi|(H-E) \psi>+<d \psi|(H-E) d \psi>}{1+2<d \psi|\psi>+<d \psi| d \psi>}
$$


which may be written

$W=E+d E+d^{2} E, \quad d E=\frac{2}{N_{0}}<d \psi\left|(H-E) \psi>, \quad d^{2} E=\frac{1}{N_{0}}<d \psi\right|(H-E) d \psi>$

with

$$
N_{0}=1+<d \psi \mid d \psi>>0
$$

where without loss of generality we may choose $d \psi$ orthogonal to $\psi$. Thus the stationary points of the functional $\mathrm{W}$ are the eigenstates of $\mathrm{H}$ :

$$
H \psi_{n}=E_{n} \psi_{n} \quad n=0,1,2 \ldots \quad E_{n}<E_{n+1}
$$

where $W-E_{n}$ is second-order and

$$
W-E_{n}=\frac{1}{N_{0}}<d \psi_{n} \mid\left(H-E_{n}\right) d \psi_{n}>
$$

If $n=0$, that is the ground state, since

$$
\Delta(m)=<\psi_{m} \mid\left(H-E_{0}\right) \psi_{m}>\quad>0 \quad m \neq 0
$$

then variations around $\psi_{0}$ give positive changes to $\mathrm{W}$ and we have a minimum principle However if we have a general $\psi_{n}$ then

$$
\Delta(m)=\left(E_{m}-E_{n}\right)<0, \quad m<n
$$

and otherwise for $m>n$ we have $\Delta(m)>0$. Thus if we choose $d \psi_{n}$ from the space spanned by the orthogonal set

$$
\left\{\psi_{m}, m=0 \ldots(n-1)\right\}
$$

then $d^{2} E<0$ and similarly choosing $d \psi_{n}$ from

$$
\left\{\psi_{m}, m=(n+1) \ldots\right\}
$$

leads to $d^{2} E>0$ (Note that this analysis is applied to a $E_{n}$ of $\mathrm{H}$ from a standard Schrödinger equation with a discrete spectrum bounded below and for which all eigenstates are square integrable). Consequently the nth eigenstate may be found from a variational principle that is not a minimum principle but a "saddle point' principle which is simultaneously a maximum 
in a space spanned by $n+1$ functions $\left\{\psi_{m}, m=0 \ldots n\right\}$ and a minimum in the space spanned by $\left\{\psi_{m}, m=n \ldots\right\}$.

However in using such a principle to estimate $\left(E_{n}, \psi_{n}\right)$ it may be more convenient to use different basis for the variations. If we choose any nonsingular operator so that

$$
\phi_{m}=U \psi_{m}
$$

then the incremental changes $d \psi_{n}$ may still be written in terms of $U^{-1} \phi_{m}=$ $\psi_{m}$. Thus the size of the subspace which generates $d^{2} E<0$ universally is unchanged and similarly for the space where $d^{2} E>0$.

Thus for any suitable chosen basis we can simultaneously maximise in the lower space and minimise in the upper space giving rise to the possibility of a variational calculation for an excited state without reference to the lower states. Conventionally one need to ensure all trial functions are independent of the lower states and this is usually done approximately by orthogonalising the trial functions to pervious calculated estimates of the lower state wave functions. Here we start with a set of functions in the domain of the Hamiltonian from which we assume the eigenfunctions form a complete set and consequently the transformed $\phi_{n}$ form a complete set. The maximisations and minimisations are carried out using a finite basis which theoretically may be expressed in terms of such a complete set.

We consider such a basis $\left\{\phi_{m}\right\}, m=0 . . N$ which depends on a set of parameters. Thus these parameters can be varied along with $\mathrm{N}$, the finite number of basis functions used, in order to refine the calculation. For any such fixed set of functions we can form the matrix $h$ and overlap s where

$$
h[i, j]=<\phi_{i}\left|H \phi_{j}>, \quad s=<\phi_{i}\right| \phi_{j}>
$$

and we solve the eigenvalue equation

$$
(h-\lambda s) \mathbf{a}=0
$$

This is equivalent to a variation of $\mathrm{W}$ subject to $\langle\psi \mid \psi\rangle=1$ where the trial function is given by

$$
\psi=\sum_{k=0}^{N} a_{k} \phi_{k}
$$

Note that we do not assume that the basis is an orthogonal basis but without loss of generality we assume that each basis element is normalised.The eigenvalues and eigenvectors, $\left(e_{k}, w_{k}\right), k=0 . . N$, found by this procedure 
are functions of the parameters in the basis elements and in particular $e_{n}$ is the maximum of the variation of $\mathrm{W}$ in the space spanned by the set $F=\left\{w_{k}, k=0 \ldots n\right\}$ and simultaneously the minimum in the space spanned by the set $G=\left\{w_{k}, k=n \ldots N\right\}$. We note that in the space generated by the set $\mathrm{F}$, since it is of dimension $n+1$, there exists at least one function $\mathrm{v}$ say, which is orthogonal to the space generated by $\left\{\psi_{m}, m=0 \ldots(n-1)\right\}$ and is therefore in the space generated by $\left\{\psi_{m}, m \geq n \ldots\right\}$. Consequently calculating $\mathrm{W}$ with $\psi=v$ gives $E(v)$ where

$$
e_{n} \geq E(v) \geq E_{n}
$$

since $e_{n}$ is the maximum value of $\mathrm{W}$ in the space generated by $\mathrm{F}$. We now vary the parameters in the basis elements and the number of basis elements $\mathrm{N}$, so that $e_{n}$ is a minimum. Note that we attain $e_{n}=E_{n}$ if $w_{k}=\psi_{k}, k=0 . . n$. This calculation is equivalent to a constrained minimisation of $\mathrm{W}$. In many calculations such a minimisation to find the nth state is carried out subject to the trial function being constrained to be orthogonal to $\mathrm{n}$ fixed functions. (These being the estimates of eigenvectors of the lower states found from previous calculations). In the calculations proposed here it is unnecessary to use any previous estimates. Furthermore since this minimisation procedure can be applied to any level of $\mathrm{h}$, then it follows that for any choice of the parameters $e_{n} \geq E_{n}$. This result is well known see for example $[1,2,3]$.

\section{A simple example}

Here we consider a set of 3 functions $u_{1}=\exp (-\alpha r), u_{2}=r \exp (-\alpha r)$, $u_{3}=r^{2} \exp (-\alpha r)$ and seek to find the $2 \mathrm{~s}$ state of atomic hydrogen by a variational calculation. The three exact lowest s states are can be expressed in terms of these functions for suitable choices of linear multiplying parameters and the non-linear exponent; a different choice of the latter is necessary for each of the three eigenstates. Thus the trial function used in the variational calculations has the form

$$
\Psi=\Psi\left(\alpha, a_{1}, a_{2}, a_{3}\right)=\sum_{k=1}^{3} a_{k} u_{k}
$$

For any fixed value of $\alpha$ we can form the $3 \times 3$ matrices $h$ and $s$ whose elements are

$$
h[n, m]=<u_{n}\left|H u_{m}>, \quad s[n, m]=<u_{n}\right| u_{m}>
$$


where the Hamiltonian is

$$
H=-\frac{1}{2} \nabla^{2}-\frac{1}{r}
$$

We choose $\lambda=E_{1}(\alpha)$ to be the second eigenvalue of the equation

$$
(h-\lambda s) \mathbf{x}=0
$$

We assume that the eigenvalues are ordered so that $E_{0}<E_{1}<E_{2}$ and denote the eigenfunctions by $w_{n}, n=0 . .2$ For this fixed value of $\alpha$ this is equivalent to a maximisation in the space spanned by $\left\{w_{0}, w_{1}\right\}$. We may simultaneously choose $\alpha$ to minimise $E_{1}$ which may be regarded as a minimisation in the space spanned by $\left\{w_{1}, w_{2}\right\}$. In the calculation here we minimise $E_{1}(\alpha)$ by choosing a sequence of values of $\alpha$ so that

$$
\alpha_{i}=\alpha_{0}+\delta(i \sqrt{2}-\operatorname{Int}[(i \sqrt{2}]), i=1 \ldots M
$$

where Int denotes the integer part. The range of the values of $\alpha_{i}$ is controlled by choices of $\alpha_{0}$ and $\delta$ and we simply choose the minimum of $E_{1}$. ( The values of $\alpha$ form an equi-distributed sequence in the interval $\alpha_{0}-\delta \ldots \alpha_{0}+\delta$.) Taking $\alpha_{0}=0, \delta=1, M=10$ leads to the estimates $E_{1}=-0.12499997, \alpha=$ 0.485281 . Another 10 iterations with $\alpha_{0}=0.485281, \delta=0.02$ leads to $E_{1}=$ $-0.1250000, \alpha=0.499839$. The eigenvalues obtained for this value of $\alpha$ are: $-0.4893339,-0.1250000,-0.01074646$ which illustrates that only the first excited state is obtained accurately and that this has been obtained without taking into account information from the lower state wave function. The wave functions for the other states cannot be represented exactly in this basis with this value of $\alpha$; for the lower state we require $\alpha=1$.The unnormalised expansion of the eigenvector for the first excited state in this basis is

$$
\phi_{1}=u_{1}-0.500161 u_{2}+0.0000804 u_{3}
$$

compared to the exact result when $\alpha=0.5$.

$$
\phi_{1}=w_{1}=u_{1}-0.5 u_{2}
$$

Similar calculations for the other two states are:

1. For the ground state calculation we obtain the eigenvalues: $-0.4999999,-0.0964758,0.79024566$ and the estimate of the ground state wave function is

$$
\phi_{0}=u_{1}-0.0 .08164 u_{2}-0.002850 u_{3} \quad \text { exact result }: u_{1}
$$


2. For the second excited state we obtain:

$-0.4339775,-0.1204813,-0.0555529$ and the estimate of the second excited state wave function is

$$
\phi_{2}=u_{1}-0.66502 u_{2}+0.07371 u_{3}, \quad \text { exact } \text { result : } u_{1}-0.66667 u_{2}+0.07407 u_{3}
$$

More precise values of the solution can easily be obtained using more iterations but the values at this stage merely illustrate the procedure.

\section{The construction of the basis for the mag- netic field problems}

The one-electron Hamiltonian for $\mathrm{H}_{2}^{+}$is the presence of a magnetic field directed along the molecular axis is is given in atomic units by

$$
H=-\frac{1}{2} \nabla^{2}-\frac{1}{r_{a}}-\frac{1}{r_{b}}+\frac{1}{R}+A L_{z}+B\left(x^{2}+y^{2}\right)
$$

where $\mathrm{R}$ is the distance between the atoms. Here we restrict the calculations to zero angular momentum so that $A=0$ and we have azimuthal symmetry. The states considered have an additional symmetry that they are symmetric or anti-symmetric with respect to a plane through mid point of the internuclear axis. We choose the origin for our calculation at this mid-point and use spheroidal coordinates :

$$
p=\frac{r_{a}+r_{b}}{R}, \quad q=\frac{r_{a}-r_{b}}{R}
$$

We choose a natural basis for this two-centred problem in the form

$$
\phi_{n}=\bar{N}_{n} L\left(n_{1}, 2 \alpha(p-1)\right) \exp (-\alpha(p-1)) P\left(m_{1}, q\right) \quad 0 \leq n_{1} \leq N_{1}, 0 \leq m_{1} \leq M_{1}
$$

where for integers $n_{1}, m_{1} L\left(n_{1}, 2 \alpha(p-1)\right)$ is a Laguerre polynomial in the variable $2 \alpha(p-1), P\left(m_{1}, q\right)$ is a Legendre polynomial and $\bar{N}_{n}$ is a normalisation constant. For symmetric states we choose $m_{1}$ even and for antisymmetric states $m_{1}$ is odd. Thus the basis depends on the single non-linear parameter, $\alpha$, which can be varied. We note that the specification of $\mathrm{H}$ by the operator in (25) is not a sufficient definition since we need to specify the 
domain of the operator. We are interested in bound states so that for all $\alpha$ the basis used is part of a complete set for square integrable wave functions, and is an appropriate set to choose.

The Hamiltonian operator for atomic hydrogen in the presence of a magnetic field

$$
-\frac{1}{2} \nabla^{2}-\frac{1}{r_{a}}+B\left(x^{2}+y^{2}\right)
$$

where again we consider states with zero orbital angular momentum and the domain of the operator is the square integrable functions. Analogous to the treatment of $\mathrm{H}_{2}^{+}$we choose a basis from a complete set that will reduce to the bound state zero field solutions as B tends to zero. For this system we choose

$$
\phi_{n}=\bar{N}_{n} L\left(n_{1}, 2 \alpha u\right) \exp (-\alpha(u)) L\left(m_{1}, 2 \alpha v\right) \exp (-\alpha(v)), \quad 0 \leq n_{1}, m_{1} \leq N_{1}
$$

where $u, v, \Phi$ are parabolic coordinates so that

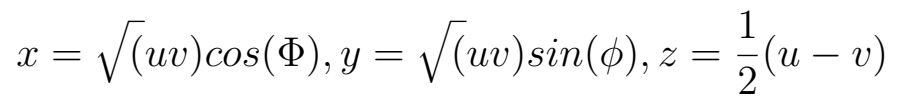

Since we are considering states of zero orbital angular momentum the basis is independent of $\Phi$. For both systems all the integrals can be evaluated analytically in terms of $\alpha$ and the field strengths considered range from $10^{8} \mathrm{G}$ which is a typical field strength from white dwarf stars to $10^{12} G$ which is level typically attained in neutron stars. In our calculations we work in atomic units and use a conversion factor $2.3505 \times 10^{9} G=1 a$.u. which differs in the last digit to that used in some of the references.

\subsection{Bound state energies for the hydrogen molecular ion in a magnetic field}

In Tables 1,2 and 3 we give the results of calculations using the functions in (27) which are obtained by choosing values $\alpha$ and the intermolecular separation $R$ and each calculation provides a bound. Partial optimisation is carried out by generating $\alpha$ using the technique described in (20) and for a sequence of values of $R$. Different approximations can be obtained by choices of $N_{1}$ and $M_{1}$ which are increased until we have consistency in the total energies to a chosen number of significant figures. We have been guided by this choice by the values in the literature that we have used for comparison. 
The wave functions can be classified as symmetric or antisymmetric with respect to plane through the mid point of the intermolecular axis and normal to this axis (the mid-point plane).Note that for symmetric wave functions the odd values of $m_{1}$ are omitted and for antisymmetric functions the even values are omitted but we have used the same number of basis functions in both variables. In Table 1 we present results for the symmetric ground state and in Table 2 the results correspond to the first excited state of symmetric functions. These calculations are completely independent and correspond to zero angular momentum so the there is azimuthal symmetry. In Table 3 we present analogous results for the lowest antisymmetric state.

Since our results are bounds we can see that they improve or agree with the comparison results. The exact position of the equilibrium internuclear distance is difficult to determine since the parameter space is flat near the minimum, but there is consistent variation of $R$ as the field strength increases. The values of $\alpha$, depend on $R, B$ and also on the size of the matrix. For a fixed matrix size these values vary consistently but become large for the larger fields, especially for the excited state. However the comparisons show that the total energies are well represented. The increase in $\alpha$ with $\mathrm{B}$ for fixed matrix size, illustrates how the optimal basis changes from one that describes an isolated molecule to one that is good for describing the field. All of the calculations can be carried out in a few minutes but for larger matrices the time taken is longer. The time is depends on the number of values of the parameters used in the optimisation, but initially partial optimisation can be carried out with a smaller basis in order to reduce the calculation time. For a fixed field strength $B$, as the size of the matrix increases the values of $\alpha$ and $R$ do not vary significantly. In the case of the excited symmetric state and $B=1 \times 10^{12}$ where the largest matrix is required, we have used only one set of values of the $\alpha$ and $R$ from a smaller matrix calculation. The gain in speed has arisen from the evaluation of the integrals which can all be done analytically compared with extensive numerical techniques which are usually required $([7],[8])$. If a different basis was used, incorporating a mixture of terms describing the field and describing the isolated molecule, then the size of the matrix might be smaller but then numerical integration would almost certainly be necessary. The eigenvalue calculations carried out here use the MAPLE programs . 
Table 1: The lowest symmetric state minima

\begin{tabular}{|c|c|c|c|c|}
$\mathrm{B}$ & $R($ a.u. $)$ & $\alpha$ & $E$ & Comparison \\
0 & 2.0 & 1.5738 & -0.60263 & $-0.60263[5]$ \\
$1 \times 10^{8}$ & 2.0 & 1.5738 & -0.60234 & $-0.60234[5]$ \\
$5 \times 10^{8}$ & 1.98 & 1.5249 & -0.59551 & $-0.59548[5]$ \\
$1 \times 10^{9}$ & 1.92 & 1.6206 & -0.57537 & $-0.57535[7]$ \\
$1 a . u$. & 1.75 & 1.8953 & -0.47498 & $-0.47496[7]$ \\
$5 \times 10^{9}$ & 1.50 & 2.1434 & -0.17478 & - \\
$1 \times 10^{10}$ & 1.25 & 2.2360 & 0.54489 & $0.54522[7]$ \\
$1 \times 10^{11}$ & 0.58 & 2.6135 & 17.52081 & $17.5216[7]$ \\
$1 \times 10^{12}$ & 0.283 & 3.4965 & 204.1499 & $204.1947[7]$
\end{tabular}

where the comparison results are taken from [5] and [7].The field strength is in Gauss and the energies in a.u.. We use the same number, $\left(N_{1}\right)$, of Laguerre functions and Legendre functions. The minimum size used is $N_{1}=4$ but for the largest field strength $N_{1}=20$ is required.

Table 2: The first excited symmetric state minima

\begin{tabular}{|c|c|c|c|c|}
$\mathrm{B}$ & $R(a . u)$ & $\alpha$ & $E$ & Comparison \\
0 & 8.8 & 3.1661 & -0.17505 & $-0.17505[5]$ \\
$1 \times 10^{8}$ & 8.8 & 3.0619 & -0.17272 & $-017270[5]$ \\
$5 \times 10^{8}$ & 8.2 & 2.5218 & -0.13384 & $-0.12939[5]$ \\
$1 \times 10^{9}$ & 7.6 & 5.0604 & -0.060792 & $-0.06062[5]$ \\
$1 a . u$. & 6.6 & 5.3946 & 0.174487 & $0.17456[7]$ \\
$5 \times 10^{9}$ & 5.8 & 6.4229 & 0.685415 & - \\
$1 \times 10^{10}$ & 5.2 & 8.0545 & 1.699210 & $1.69969[7]$ \\
$1 \times 10^{11}$ & 3.9 & 17.7582 & 20.70149 & $20.7045[7]$ \\
$1 \times 10^{12}$ & 3.55 & 80.6912 & 212.1062 & $212.11385[7]$
\end{tabular}

where the comparison results are taken from [5] and [7]. The field strength is in Gauss and the energies in a.u..We use the same number, $\left(N_{1}\right)$, of Laguerre functions and Legendre functions. The minimum size used is $N_{1}=4$ but for the largest field strength $N_{1}=49$ is required. 
Table 3: The lowest antisymmetric state minima

\begin{tabular}{|c|c|c|c|c|}
$\mathrm{B}$ & $R(a . u)$ & $\alpha$ & $E$ & Comparison \\
0 & 12.55 & 6.24654 & -0.500061 & $-0.500061[5]$ \\
$1 \times 10^{8}$ & 12.55 & 6.24654 & -0.499610 & $-0.499610[5]$ \\
$5 \times 10^{8}$ & 12.00 & 7.68703 & -0.489251 & $-0.489050[5]$ \\
$1 \times 10^{9}$ & 11.5 & 7.6031 & -0.460563 & $-0.460515[7]$ \\
1 a.u. & 9.58 & 10.43018 & -0.3314365 & $-0.3314365[8]$ \\
$5 \times 10^{9}$ & 8.4 & 10.5749 & 0.020852 & $0.10097[5]$ \\
$1 \times 10^{10}$ & 7.0 & 11.72655 & 0.8191167 & $0.81995[7]$ \\
$1 \times 10^{11}$ & 5.0 & 28.47712 & 18.41176 & $18.41835[7]$ \\
$1 \times 10^{12}$ & 3.2 & 50.051336 & 206.7727 & $206.8038[7]$
\end{tabular}

where the comparison results are taken from [5],[7] and [8].The field strength is in Gauss and the energies in a.u.. We use the same number, $\left(N_{1}\right)$, of Laguerre functions and Legendre functions. The minimum size used is $N_{1}=5$ but for the largest field strength $N_{1}=35$ is required.

To improve the efficiency of the calculation we have chosen to construct smaller matrices in order to set up the Hamiltonian matrix. For $\mathrm{H}_{2}^{+}$these are:

$$
L P[k]=\int_{1}^{\infty} L\left(n_{1}, 2 \alpha(p-1)\right) p^{k} L\left(n_{2}, 2 \alpha(p-1) \exp (-2 \alpha(p-1)) d p\right.
$$

of size $N_{1} \times N_{1}$. Similarly the moments of q, LQ $[\mathrm{k}]$,can be calculated using the functions $P_{m}(q)$ leading to matrices of size $M_{1} \times M_{1}$. To treat the Kinetic energy terms we need two extra matrices, TP and TQ, of size $N_{1} \times N_{1}$ and $M_{1} \times M_{1}$ respectively. The matrix TQ is simply a diagonal matrix whose diagonal elements are determined from the Legendre differential equation and the elements of TP may be calculated in the basis in (27) where additionally the generalised Legendre polynomial $L(n, 1, x)$ is used for the derivative terms. All the Legendre functions are available in MAPLE. The matrix elements are functions of $\alpha$ and for any given numerical value of this parameter the matrices can be used to evaluate the $N_{1} M_{1} \times N_{1} M_{1}$ matrix elements of the total Hamiltonian. For example for a given $\alpha$

$$
<\phi_{i} \mid\left(\frac{1}{r_{a}}+\frac{1}{r_{b}}\right) \phi_{j}>=K_{i} K_{j} L P[1]\left[n_{1}, n_{2}\right] L Q[0]\left[m_{1}, m_{2}\right]
$$


where $K_{i}, K_{j}$ depend on the normalisation, $i$ is associated with the pair $\left(n_{1}, m_{1}\right)$ and $j$ with $\left(n_{2}, m_{2}\right)$. Thus the size of the calculation has been reduced from $O\left(N_{1}^{2} M_{2}^{2}\right)$ to $O\left(\hat{N}^{2}\right)$ where $\hat{N}$ is the maximum of $N_{1}$ and $M_{1}$.

The minimisation procedure used is as is described in (20) where $\sqrt{2}$ may be replaced by $\sqrt{P_{r}}$ for any prime number $P_{r}$. The range of values required in the calculations with large fields can be pre-determined by less accurate calculations with a smaller basis.

\subsection{Bound state energies for hydrogen in a magnetic field}

In parabolic coordinates (28) becomes

$$
H=-\frac{1}{2} \nabla^{2}-\frac{2}{u+v}+B u v
$$

Since this is symmetric in the interchange of $u$ and $v$, then, provided there is no degeneracy, the eigenfunctions are either symmetric or antisymmetric in the interchange of $u$ and $v$. (For degenerate eigenfunctions, they can be chosen symmetric or antisymmetric in this interchange). Thus in the limit as $B$ tends to 0 we obtain s and $\mathrm{p}$ states respectively; but in this case there is degeneracy. This simplifies the calculation since rather than the full basis described in (29), we may use

$$
\begin{gathered}
\phi_{n}=\bar{N}_{n} L\left(n_{1}, 2 \alpha u\right) \exp (-\alpha(u)) L\left(m_{1}, 2 \alpha v\right) \exp (-\alpha(v)) \pm \\
\bar{N}_{n} L\left(n_{1}, 2 \alpha u\right) \exp (-\alpha(u)) L\left(m_{1}, 2 \alpha v\right) \exp (-\alpha(v)), \quad n_{1} \geq m_{1}
\end{gathered}
$$

with + used for symmetric states and - for antisymmetric states. With these choices the sizes of the matrices are reduced from $N_{1}^{2}$ to $\left(N_{1}+1\right)\left(N_{1}+2\right) / 2$ or $N_{1}\left(N_{1}+1\right) / 2$ respectively. In tables 4,5 , and 6 we present results for the two lowest symmetric states and the lowest antisymmetric state.For a fixed basis size the value of $\alpha$ decreases as the field strength increases and the results are consistent with the comparisons available. In tables 4 and 5, where more comparison results are available, we increase the size of the basis to match the results in the literature. In table 6 the only comparisons used are with [5] which are not bounds. However our results, which are bounds, are consistent with the values in [5]. We conclude that the variational scheme proposed here is useful even for more difficult problems such as the application of a large 
magnetic field. One big advantage is that the calculation for each state can be done without reference to the other states. The calculations presented here are carried out using analytical integration and this is one of the main reasons that they do not take excessive time.

\section{Conclusion}

We have adapted a well known variational method so as to obtain bounds for each state independently of the other states and applied this to the problems of hydrogen and the hydrogen molecular ion in weak and strong magnetic fields. To do this we use a basis taken from complete sets in the space. Each of these sets contains an arbitrary positive parameter, $\alpha$. The basis is chosen by truncating the expansion in a particular set and choosing $\alpha$ as a variational parameter for one particular state. The variational estimate of the energy is then found by optimising the appropriate eigenstate of the secular equation in this basis. In each case we have chosen a separable basis which reduces the number of matrix element calculations required and all integrations may be evaluated analytically in terms of $\alpha, \mathrm{B}$ and $\mathrm{R}$ so that the same elements can be used for different problems. For weak fields the secular equation calculation converges for small-sized matrices but for the stronger fields larger matrices are required in order to obtain at least 5 digit accuracy in the comparisons. More qualitative results to less than 1 precent accuracy can be obtained with much smaller matrices but for all the calculations carried out here the Maple routines were sufficient. In general a more sophisticated choice of basis with more variational parameters may result in smaller matrices for a given accuracy, but this will almost certainly require numerical integration for which there will be additional problems of accuracy and the time taken for each calculation.

\section{References}

[1] Eckart C E 1930 Phys Rev 36878

[2] Hylleras E, and Underheim B 1930,Z.Phys. 65759

[3] McDonald J K L 1933 Phys. Rev 43830

[4] Xiong Z, Bacalis N C and Karaaoulanis D 2008 J.Comp.Meth.Sci.Eng 8 277 
[5] Peek J M and Katriel J 1980 Phys. Rev A 21413

[6] Cohen M and Kais S 1984 J Phys A 173049

[7] Turbiner A V and Lopez J C 2004 Phys Rev A 69053413

[8] Guan X,Li B and Taylor K T 2003 J Phys B 363569

[9] Lopez J C and Pilon H O 2008 Revista Mexico.de Fisica 5449

Table 4: The lowest symmetric state minima for $\mathrm{H}$

\begin{tabular}{|c|c|c|c|c|}
$\mathrm{B}$ & $\alpha$ & $E$ & Comparison & Comparison \\
$1 \times 10^{8}$ & 5.12409 & -0.499548 & $-0.499548[5]$ & - \\
0.1 a.u. & 2.4655 & -0.4975265 & $-0.4975265[9]$ & $-0.49753[6]$ \\
$1 \times 10^{9}$ & 1.9685 & -0.460427 & $-0.4604113[9]$ & - \\
1 a.u & 1.95252 & -0.3311689 & $-0.331166[9]$ & $-0.33052[6]$ \\
$1 \times 10^{10}$ & 1.76349. & 0.819733 & $0.820181[9]$ & - \\
$10 a . u$. & 1.637226 & 3.252203 & $3.25261[9]$ & $3.2575[6]$ \\
$1 \times 10^{11}$ & 1.49617 & 18.41261 & $18.4199[9]$ & - \\
$100 a . u$ & 1.4439 & 46.211 & $46.2178[9]$ & $46.275[6]$ \\
$1 \times 10^{12}$ & 1.389027 & 206.814439 & $206.831[9]$ & -
\end{tabular}

where the comparison results are taken from [5] [6] and [9].The field strength is in Gauss $\left(1 . a . u=2.3505 \times 10^{9} G\right)$ and the energies in a.u.The minimum number of Laguerre functions used is 4 but for the largest field strength 15 Laguerre functions are required. 
Table 5: The lowest antisymmetric state minima for $\mathrm{H}$

$$
\begin{array}{|c|c|c|c|}
\mathrm{B} & \alpha & E & \text { Comparison } \\
1 \times 10^{8} & 4.1246 & -0.122403 & -0.12237[5] \\
0.1 a . u . & 2.96190 & -0.1124100 & -0.1124099[9] \\
1 \times 10^{9} & 1.860171 & -0.00439568 & -0.004351[9] \\
1 a . u & 1.6833996 & 0.23999339 & 0.24004[9] \\
1 \times 10^{10} & 1.64 & 1.7881937 & 1.78895[9] \\
10 a . u . & 1.63 & 4.61735 & 4.61795[9] \\
1 \times 10^{11} & 1.63 & 20.83136 & 20.837[9] \\
100 a . u & 1.63 & 49.5367 & 49.537[9] \\
1 \times 10^{12} & 1.63 & 212.28 & 212.28[9]
\end{array}
$$

where the comparison results are taken from [5] and [9]. The field strength is in Gauss $\left(1 a . u=2.3505 \times 10^{9} G\right.$ ) and the energies in a.u.The minimum number of Laguerre functions used is 9 but for the largest field strength 71 Laguerre functions are required.

Table 6: The first excited symmetric state minima for $\mathrm{H}$

$$
\begin{array}{|c|c|c|c|}
\mathrm{B} & \alpha & E & \text { Comparison } \\
1 \times 10^{8} & 2.682 & -0.119091 & -0.1207[5] \\
0.1 a . u . & 1.999 & -0.098089 & - \\
1 \times 10^{9} & 1.53 & 0.063190 & 0.06080[5] \\
1 a . u & 1.39 & 0.339642 & - \\
1 \times 10^{10} & 1.40 & 1.938609 & - \\
10 a . u . & 1.36 & 4.80949 & - \\
1 \times 10^{11} & 1.33 & 21.3254 & - \\
100 a . u & 1.31 & 162.4052 & - \\
1 \times 10^{12} & 1.31 & 218.9834 & -
\end{array}
$$

where the comparison results are taken from [5].The field strength is in Gauss $\left(1 . a . u=2.3505 \times 10^{9} G\right)$ and the energies in a.u. The number of Laguerre functions used in all of the calculations is 15 . 